\title{
What's in a Name?
}

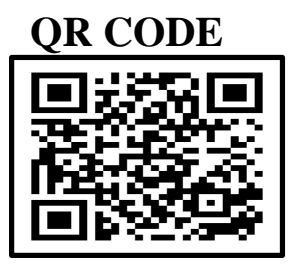

\section{MANISHA LAKHANPAL SHARMA ${ }^{\text {ID }}$}

Oral Medicine and Radiology (OMDR) is a discipline of dentistry concerned with the diagnosis and management of conditions that affect the oral and maxillofacial region. In any institute, it is the first department which interacts with the patient, chalks out a detailed treatment plan and garners confidence to the patient. Paradoxically, it is referred to as a nonclinical branch, the rationale of which being that, an oral physician dispenses only medicinal treatment to the patient. This logic is strange as an MD medicine in the same capacity does not belong to a non-clinical branch nor does MD radiology. On the contrary, these branches are highly valued by the aspiring candidates. The beauty of OMDR is an unexplored territory as this department has been reduced to a referral center by many. Lately, in dentistry, there has been a paradigm shift from a diagnosis and treatment centric approach to just treatment dispensing approach. This shadows the importance of not only correct diagnosis but also the underlying systemic health of an individual as well as the incidental findings. Therefore, there are many instances of treatment related complications, the worst being initiation of infiltration in a cancer patient undergoing extractions without being diagnosed or application of corticosteroid on a non-healing tubercular ulcer. Oral medicine specialists do not deal with cadavers, slides, pipettes; rather they deal with patients suffering from devastating orofacial pain, premalignant lesions and conditions, diagnosis of malignancies, temporomandibular disorders, salivary gland diseases, oral manifestations of systemic diseases and the list goes on. Not only this, their eyes are trained to catch a variation in the grey scale on a radiograph depicting abnormality like no other branch. The advent of Cone Beam Computed Tomography (CBCT) has further elevated the field by adding a zing to $3 \mathrm{D}$ radiology. The advent of technology like LASER aids the branch to broaden the plethora of treatments starting from eradication of potentially malignant disorders to operculectomy. Now -a- days many institutes are stressing upon the concept of comprehensive clinical approach towards a patient. This gives an opportunity to the postgraduates to conduct the basic treatment modalities for the patient whether healthy or immunocompromised. The title non clinical somehow dampens the potential of the branch and dissuades candidates from selecting it as a postgraduate course. It is rightly said that "Words have meaning but titles have power". In this case, the title does not justify the power of the branch. 